\title{
Attractivity for a $k$-dimensional system of fractional functional differential equations and global attractivity for a $k$-dimensional system of nonlinear fractional differential equations
}

\author{
Dumitru Baleanu ${ }^{1,2,3^{*}}$, Sayyedeh Zahra Nazemi ${ }^{4}$ and Shahram Rezapour ${ }^{4}$
}

${ }^{*}$ Correspondence:

dumitru@cankaya.edu.tr

'Department of Mathematics,

Cankaya University, Ogretmenler

Cad. 14, Balgat, Ankara, 06530,

Turkey

${ }^{2}$ Institute of Space Sciences,

Magurele, Bucharest, Romania

Full list of author information is

available at the end of the article

\begin{abstract}
In this paper, we present some results for the attractivity of solutions for a $k$-dimensional system of fractional functional differential equations involving the Caputo fractional derivative by using the classical Schauder's fixed-point theorem. Also, the global attractivity of solutions for a $k$-dimensional system of fractional differential equations involving Riemann-Liouville fractional derivative are obtained by using Krasnoselskii's fixed-point theorem. We give two examples to illustrate our main results.
\end{abstract}

\section{Introduction}

In recent years, many researchers have been focused on investigation of fractional differential equations which has played an important role in different areas of science (see for example, [1-22] and the references therein). As you know, there are many practical applications of fractional differential equations in different fields of science such as economy, biology, and the study of forced van der Pol oscillators (see for example, [23-25] and the references therein). On the other hand, there are a few papers on the attractivity of solutions for fractional differential equations and fractional functional differential equations (see for example, [15] and [16]). For the details of basic notions of this paper such the standard Caputo fractional derivative, the standard Riemann-Liouville fractional derivative, and the fractional integral of order $q$ for a function $f$ see [18]. In 2011, Chen and Zhou reviewed the attractivity of solutions for the fractional functional differential equation ${ }^{c} D^{\alpha} x(t)=f\left(t, x_{t}\right)$ for $t \in\left(t_{0}, \infty\right)$ via the boundary value condition $x(t)=\phi(t)$ for $t_{0}-r \leq t \leq t_{0}$, where $t_{0} \geq 0, r>0,0<\alpha<1,{ }^{c} D$ is the standard Caputo fractional derivative, $\phi \in C\left(\left[t_{0}-r, t_{0}\right], \mathbb{R}\right)$ and $f:\left(t_{0}, \infty\right) \times C([-r, 0], \mathbb{R}) \rightarrow \mathbb{R}$ a function with some properties [16]. In 2012, Chen et al. reviewed the global attractivity of solutions for the nonlinear fractional differential equation $D^{\alpha} x(t)=g(t, x(t))$ for $t \in\left(t_{0}, \infty\right)$ via the boundary value problem $\left[D^{\alpha-1} x(t)\right]_{t=t_{0}}=x_{0}$, where $t_{0} \geq 0,0<\alpha<1, x_{0}$ is a constant, $D$ is the standard Riemann-Liouville fractional derivative, and $g:\left(t_{0}, \infty\right) \times \mathbb{R} \rightarrow \mathbb{R}$ a function with some properties [15]. Also, they investigated the global attractivity of solutions for the nonlinear fractional differential equation ${ }^{c} D^{\alpha} x(t)=g(t, x(t))$ for $t \in\left(t_{0}, \infty\right)$ via the boundary value 
problem $x\left(t_{0}\right)=x_{0}$, where $t_{0} \geq 0,0<\alpha<1, x_{0}$ is a constant, ${ }^{c} D$ is the standard Caputo fractional derivative, and $g:\left(t_{0}, \infty\right) \times \mathbb{R} \rightarrow \mathbb{R}$ a function with some properties [15].

In this paper, we investigate the attractivity of solutions for a $k$-dimensional system of fractional differential equations. Also, we investigate the global attractivity of solutions for another $k$-dimensional system of nonlinear fractional differential equations.

\section{Preliminaries}

In this paper, we investigate the attractivity of solutions for $k$-dimensional system of fractional functional differential equations

$$
\left\{\begin{array}{l}
{ }^{c} D^{\alpha_{1}} x_{1}(t)=f_{1}\left(t, x_{1_{t}}, x_{2_{t}}, \ldots, x_{k_{t}}\right), \\
{ }^{c} D^{\alpha_{2}} x_{2}(t)=f_{2}\left(t, x_{1_{t}}, x_{2 t}, \ldots, x_{k_{t}}\right), \\
\vdots \\
{ }^{c} D^{\alpha_{k}} x_{k}(t)=f_{k}\left(t, x_{1_{t}}, x_{2_{t}}, \ldots, x_{k_{t}}\right),
\end{array}\right.
$$

via the boundary value problems $x_{1}(t)=\phi_{1}(t), x_{2}(t)=\phi_{2}(t), \ldots, x_{k}(t)=\phi_{k}(t)$ for $t_{0}-r \leq$ $t \leq t_{0}$, where $0<\alpha_{i}<1$ for $i=1,2, \ldots, k, t_{0} \geq 0, t \in\left(t_{0}, \infty\right),{ }^{c} D$ is the standard Caputo fractional derivative, $J=\left(t_{0}, \infty\right), r>0, \phi_{i} \in C\left(\left[t_{0}-r, t_{0}\right], \mathbb{R}^{n}\right)$ for $i=1,2, \ldots, k$ and $f_{i}: J \times$ $C\left([-r, 0], \mathbb{R}^{n}\right) \times C\left([-r, 0], \mathbb{R}^{n}\right) \times \cdots \times C\left([-r, 0], \mathbb{R}^{n}\right) \rightarrow \mathbb{R}^{n}$ is a function satisfying some assumptions that will be specified later for $i=1,2, \ldots, k$. If $x \in C\left(\left[t_{0}-r, \infty\right), \mathbb{R}^{n}\right)$, then $x_{t}$ is defined by $x_{t}(\theta)=x(t+\theta)$ for $-r \leq \theta \leq 0$ and $t \in\left[t_{0}, \infty\right)$. Also, we investigate the global attractivity of solutions for the $k$-dimensional system of nonlinear fractional differential equations

$$
\left\{\begin{array}{l}
D^{\alpha_{1}} x_{1}(t)=g_{1}\left(t, x_{1}(t), x_{2}(t), \ldots, x_{k}(t)\right), \\
D^{\alpha_{2}} x_{2}(t)=g_{2}\left(t, x_{1}(t), x_{2}(t), \ldots, x_{k}(t)\right), \\
\vdots \\
D^{\alpha_{k}} x_{k}(t)=g_{k}\left(t, x_{1}(t), x_{2}(t), \ldots, x_{k}(t)\right),
\end{array}\right.
$$

via the boundary value problems $\left[D^{\alpha_{1}-1} x_{1}(t)\right]_{t=t_{0}}=x_{1}^{0},\left[D^{\alpha_{2}-1} x_{2}(t)\right]_{t=t_{0}}=x_{2}^{0}, \ldots,\left[D^{\alpha_{k}-1} \times\right.$ $\left.x_{k}(t)\right]_{t=t_{0}}=x_{k}^{0}$, where $0<\alpha_{i}<1$ for $i=1,2, \ldots, k, t_{0} \geq 0, t \in\left(t_{0}, \infty\right), D$ is the RiemannLiouville fractional derivative, $J=\left(t_{0}, \infty\right), x_{1}^{0}, \ldots, x_{k}^{0}$ are constants, and $g_{i}: J \times \mathbb{R}^{n} \times \mathbb{R}^{n} \times$ $\cdots \times \mathbb{R}^{n} \rightarrow \mathbb{R}^{n}$ is an integrable function satisfying some assumptions that will be specified later for $i=1,2, \ldots, k$. In fact, we say that the solution $\left(x_{1}(t), x_{2}(t), \ldots, x_{k}(t)\right)$ of the problem (2.1) is attractive whenever if there exists a constant $b_{i}^{0}\left(t_{0}\right)>0$ such that $\left|\phi_{i}(s)\right| \leq b_{i}^{0}$ for all $i=1,2, \ldots, k$ and $s \in\left[t_{0}-r, t_{0}\right]$, then $\lim _{t \rightarrow \infty} x_{i}\left(t, t_{0}, \phi_{i}\right) \rightarrow 0$. Also, the zero solution $x(t)$ of the problem (2.2) is said to be globally attractive whenever each solution tends to zero as $t \rightarrow \infty$. Let $X=C\left(J, \mathbb{R}^{n}\right)$ be the Banach space of all continuous functions from $J$ into $\mathbb{R}^{n}$ with the norm $\|x\|=\sup _{t \in J}|x(t)|$, where $|\cdot|$ denotes a suitable complete norm on $\mathbb{R}^{n}$. It is clear that the product space $(X^{k}=\underbrace{X \times X \times \cdots \times X}_{k},\|\cdot\|_{*})$ is also a Banach space, where $\left\|\left(x_{1}, x_{2}, \ldots, x_{k}\right)\right\|_{*}=\left\|x_{1}\right\|+\left\|x_{2}\right\|+\cdots+\left\|x_{k}\right\|$. We need the following Schauder fixed-point theorem and improvement of a fixed-point theorem of Krasnoselskii due to Burton, which one can find in $[14,17]$ and [19].

Theorem 2.1 If $U$ is a nonempty, closed, bounded, and convex subset of the Banach space $X$ and $T: U \rightarrow U$ is completely continuous, then $T$ has a fixed point. 
Theorem 2.2 Let $S$ be a nonempty, closed, convex, and bounded subset of the Banach space $X, A: X \rightarrow X$ a contraction with constant $l<1, B: S \rightarrow X$ a continuous map which $B(S)$ resides in a compact subset of $X$ and $x=A x+B y$ and $y \in S$ implies $x \in S$. Then the operator equation $A x+B x=x$ has a solution in $S$.

\section{Main results}

First, we investigate attractive solutions of the problem (2.1). In this way, suppose that $\left\|x_{t}\right\|=\sup _{-r \leq \theta \leq 0}|x(t+\theta)|$ for $t \in J$. We assume that $f_{i}\left(t, x_{1_{t}}, x_{2_{t}}, \ldots, x_{k_{t}}\right)$ is Lebesgue measurable with respect to $t$ on $\left[t_{0}, \infty\right)$ and $f_{i}\left(t, \varphi_{1}, \varphi_{2}, \ldots, \varphi_{k}\right)$ is continuous with respect to $\varphi_{j}$ on $C\left([-r, 0], \mathbb{R}^{n}\right)$ for $i, j=1,2, \ldots, k$. Note that the problem (2.1) is equivalent to the system of equations

$$
x_{i}(t)= \begin{cases}\phi_{i}\left(t_{0}\right)+\frac{1}{\Gamma\left(\alpha_{i}\right)} \int_{t_{0}}^{t}(t-s)^{\alpha_{i}-1} f_{i}\left(s, x_{1_{s}}, x_{2_{s}}, \ldots, x_{k_{s}}\right) d s, & t>t_{0}, \\ \phi_{i}(t), & t \in\left[t_{0}-r, t_{0}\right]\end{cases}
$$

or

$$
x_{i}(t)= \begin{cases}\frac{1}{\Gamma\left(\alpha_{i}\right)} \int_{t_{0}}^{t}(t-s)^{\alpha_{i}-1}\left[\frac{\phi_{i}\left(t_{0}\right)}{\Gamma\left(1-\alpha_{i}\right)}\left(s-t_{0}\right)^{-\alpha_{i}}+f_{i}\left(s, x_{1_{s}}, x_{2_{s}}, \ldots, x_{k_{s}}\right)\right] d s, & t>t_{0}, \\ \phi_{i}(t), & t \in\left[t_{0}-r, t_{0}\right]\end{cases}
$$

for $i=1,2, \ldots, k$. Define the operator $T: X^{k} \rightarrow X^{k}$ by

$$
T\left(x_{1}, x_{2}, \ldots, x_{k}\right)(t)=\left(\begin{array}{l}
T_{1}\left(x_{1}, x_{2}, \ldots, x_{k}\right)(t) \\
T_{2}\left(x_{1}, x_{2}, \ldots, x_{k}\right)(t) \\
\vdots \\
T_{k}\left(x_{1}, x_{2}, \ldots, x_{k}\right)(t)
\end{array}\right),
$$

where

$$
T_{i}\left(x_{1}, x_{2}, \ldots, x_{k}\right)(t)= \begin{cases}\phi_{i}\left(t_{0}\right)+\frac{1}{\Gamma\left(\alpha_{i}\right)} \int_{t_{0}}^{t}(t-s)^{\alpha_{i}-1} f_{i}\left(s, x_{1_{s}}, x_{2_{s}}, \ldots, x_{k_{s}}\right) d s, & t>t_{0}, \\ \phi_{i}(t), & t \in\left[t_{0}-r, t_{0}\right]\end{cases}
$$

for $i=1,2, \ldots, k$. It is easy to check that $\left(x_{1}(t), x_{2}(t), \ldots, x_{k}(t)\right)$ is a solution of the problem (2.1) if and only if $\left(x_{1}(t), x_{2}(t), \ldots, x_{k}(t)\right)$ is a fixed point of the operator $T$.

Theorem 3.1 Suppose that for each $i \in\{1,2, \ldots, k\}$ there exist $\gamma_{i 1}>0$ and $\alpha_{i 1} \in\left(0, \alpha_{i}\right)$ such that

$$
\left|\phi_{i}\left(t_{0}\right)+\frac{1}{\Gamma\left(\alpha_{i}\right)} \int_{t_{0}}^{t}(t-s)^{\alpha_{i}-1} f_{i}\left(s, x_{1_{s}}, x_{2_{s}}, \ldots, x_{k_{s}}\right) d s\right| \leq\left(t-t_{0}\right)^{-\gamma_{i 1}}
$$

for all $t \in J$ and $f_{i} \in L^{\frac{1}{\alpha_{i 1}}}\left(J \times C\left([-r, 0], \mathbb{R}^{n}\right) \times C\left([-r, 0], \mathbb{R}^{n}\right) \times \cdots \times C\left([-r, 0], \mathbb{R}^{n}\right), \mathbb{R}^{n}\right)$. Then the problem (2.1) has at least one attractive solution $\left(x_{1}, x_{2}, \ldots, x_{k}\right)$ such that $x_{i} \in C\left(\left[t_{0}-\right.\right.$ $r, \infty), \mathbb{R}^{n}$ ) for all $i=1,2, \ldots, k$.

Proof Consider the set

$$
\begin{aligned}
S_{1}= & \left\{\left(x_{1}, x_{2}, \ldots, x_{k}\right): x_{i} \in C\left(\left[t_{0}-r, \infty\right), \mathbb{R}^{n}\right),\left|x_{i}(t)\right| \leq\left(t-t_{0}\right)^{-\gamma_{i 1}}\right. \\
& \left.\quad \text { for all } i=1,2, \ldots, k \text { and } t \geq \tilde{t}>t_{0}\right\},
\end{aligned}
$$


where $\tilde{t}$ is a constant. It is easy to check that $S_{1}$ is a closed, bounded, and convex subset of $\underbrace{\mathbb{R}^{n} \times \mathbb{R}^{n} \times \cdots \times \mathbb{R}^{n}}_{k}$. We show that the operator $T$ has a fixed point in $S_{1}$. This implies that the problem (2.1) has a solution. Note that $\left|T_{i}\left(x_{1}, x_{2}, \ldots, x_{k}\right)(t)\right| \leq(t-$ $\left.t_{0}\right)^{-\gamma_{i 1}}$ for all $i=1,2, \ldots, k$ and so $T\left(S_{1}\right) \subset S_{1}$. Now, we show that $T$ is continuous. Let $\left(x_{1}^{m}, x_{2}^{m}, \ldots, x_{k}^{m}\right),\left(x_{1}, x_{2}, \ldots, x_{k}\right) \in S_{1}$ for all $m \geq 1$ and $\lim _{m \rightarrow \infty}\left|x_{i}^{m}(t)-x_{i}(t)\right|=0$ for all $i=1,2, \ldots, k$. Then, we have $\lim _{m \rightarrow \infty} f_{i}\left(t, x_{1_{t}}^{m}, x_{2_{t}}^{m}, \ldots, x_{k_{t}}^{m}\right)=f_{i}\left(t, x_{1_{t}}, x_{2_{t}}, \ldots, x_{k_{t}}\right)$ for all $i=$ $1,2, \ldots, k$ and $t>t_{0}$. Let $\epsilon>0$ be given. Choose $\tilde{T}>t_{0}$ such that $t \geq \tilde{T}$ implies that $\left(t-t_{0}\right)^{-\gamma_{i 1}}<\frac{\epsilon}{2}$. Let $v_{i 1}=\frac{\alpha_{i}-1}{1-\alpha_{i 1}}$ and note that $1+v_{i 1}>0$ for $i=1,2, \ldots, k$. Also, we have

$$
\begin{aligned}
\left|T_{i}\left(x_{1}^{m}, x_{2}^{m}, \ldots, x_{k}^{m}\right)(t)-T_{i}\left(x_{1}, x_{2}, \ldots, x_{k}\right)(t)\right| \\
\leq \frac{1}{\Gamma\left(\alpha_{i}\right)} \int_{t_{0}}^{t}(t-s)^{\alpha_{i}-1}\left|f_{i}\left(s, x_{1_{s}}^{m}, x_{2_{s}}^{m}, \ldots, x_{k_{s}}^{m}\right)-f_{i}\left(s, x_{1_{s}}, x_{2_{s}}, \ldots, x_{k_{s}}\right)\right| d s \\
\leq \frac{1}{\Gamma\left(\alpha_{i}\right)}\left\{\int_{t_{0}}^{t}\left[(t-s)^{\alpha_{i}-1}\right]^{\frac{1}{1-\alpha_{i 1}}} d s\right\}^{1-\alpha_{i 1}} \\
\quad \times\left[\int_{t_{0}}^{t} \mid f_{i}\left(s, x_{1_{s}}^{m}, x_{2_{s}}^{m}, \ldots, x_{k_{s}}^{m}\right)-f_{i}\left(s, x_{1_{s}}, x_{2_{s}}, \ldots, x_{k_{s}}\right)^{\frac{1}{\alpha_{i 1}}} d s\right]^{\alpha_{i 1}} \\
\leq \frac{1}{\Gamma\left(\alpha_{i}\right)}\left(\frac{1}{1+v_{i 1}}\left(t-t_{0}\right)^{1+v_{i 1}}\right)^{1-\alpha_{i 1}} \\
\quad \times\left[\int_{t_{0}}^{\tilde{T}}\left|f_{i}\left(s, x_{1_{s}}^{m}, x_{2_{s}}^{m}, \ldots, x_{k_{s}}^{m}\right)-f_{i}\left(s, x_{1_{s}}, x_{2_{s}}, \ldots, x_{k_{s}}\right)\right|^{\frac{1}{\alpha_{i 1}}} d s\right]^{\alpha_{i 1}} \\
\leq \frac{1}{\Gamma\left(\alpha_{i}\right)}\left(\frac{1}{1+v_{i 1}}\left(\tilde{T}-t_{0}\right)^{1+v_{i 1}}\right)^{1-\alpha_{i 1}}\left(\tilde{T}-t_{0}\right)^{\alpha_{i 1}} \\
\quad \times \sup _{t_{0} \leq s \leq \tilde{T}}\left|f_{i}\left(s, x_{1_{s}}^{m}, x_{2_{s}}^{m}, \ldots, x_{k_{s}}^{m}\right)-f_{i}\left(s, x_{1_{s}}, x_{2_{s}}, \ldots, x_{k_{s}}\right)\right|
\end{aligned}
$$

for $t_{0}<t \leq \tilde{T}$. Thus, $\lim _{m \rightarrow \infty}\left|T_{i}\left(x_{1}^{m}, x_{2}^{m}, \ldots, x_{k}^{m}\right)(t)-T_{i}\left(x_{1}, x_{2}, \ldots, x_{k}\right)(t)\right|=0$ for all $t_{0}<$ $t \leq \tilde{T}$. Also, we have

$$
\begin{aligned}
& \left|T_{i}\left(x_{1}^{m}, x_{2}^{m}, \ldots, x_{k}^{m}\right)(t)-T_{i}\left(x_{1}, x_{2}, \ldots, x_{k}\right)(t)\right| \\
& =\mid \frac{1}{\Gamma\left(\alpha_{i}\right)} \int_{t_{0}}^{t}(t-s)^{\alpha_{i}-1} f_{i}\left(s, x_{1_{s}}^{m}, x_{2_{s}}^{m}, \ldots, x_{k_{s}}^{m}\right) d s \\
& \quad-\frac{1}{\Gamma\left(\alpha_{i}\right)} \int_{t_{0}}^{t}(t-s)^{\alpha_{i}-1} f_{i}\left(s, x_{1_{s}}, x_{2_{s}}, \ldots, x_{k_{s}}\right) d s \mid \\
& \quad \leq 2\left(t-t_{0}\right)^{-\gamma_{i 1}} \leq \epsilon
\end{aligned}
$$

for $t>\tilde{T}$. Hence, $\lim _{m \rightarrow \infty}\left|T_{i}\left(x_{1}^{m}, x_{2}^{m}, \ldots, x_{k}^{m}\right)(t)-T_{i}\left(x_{1}, x_{2}, \ldots, x_{k}\right)(t)\right|=0$ for $t>t_{0}$. This implies that $T_{i}$ is continuous for $i=1,2, \ldots, k$ and so $T$ is continuous. Now, we show that the set $T\left(S_{1}\right)$ is equi-continuous. Let $\epsilon>0$. Since $\lim _{t \rightarrow \infty}\left(t-t_{0}\right)^{-\gamma_{i 1}}=0$ for $i=1,2, \ldots, k$, there is a $\tilde{T}^{\prime}>t_{0}$ such that $\left(t-t_{0}\right)^{-\gamma_{i 1}}<\frac{\epsilon}{2}$ for all $t>\tilde{T}^{\prime}$ and $i=1,2, \ldots, k$. Let $t_{1}, t_{2}>t_{0}$ and $t_{2}>t_{1}$. If $t_{1}, t_{2} \in\left(t_{0}, \tilde{T}^{\prime}\right]$, then

$$
\begin{aligned}
& \left|T_{i}\left(x_{1}, x_{2}, \ldots, x_{k}\right)\left(t_{2}\right)-T_{i}\left(x_{1}, x_{2}, \ldots, x_{k}\right)\left(t_{1}\right)\right| \\
& \quad \leq \mid \frac{1}{\Gamma\left(\alpha_{i}\right)} \int_{t_{0}}^{t_{2}}\left(t_{2}-s\right)^{\alpha_{i}-1} f_{i}\left(s, x_{1_{s}}, x_{2_{s}}, \ldots, x_{k_{s}}\right) d s
\end{aligned}
$$




$$
\begin{aligned}
& -\frac{1}{\Gamma\left(\alpha_{i}\right)} \int_{t_{0}}^{t_{1}}\left(t_{1}-s\right)^{\alpha_{i}-1} f_{i}\left(s, x_{1_{s}}, x_{2_{s}}, \ldots, x_{k_{s}}\right) d s \mid \\
\leq & \frac{1}{\Gamma\left(\alpha_{i}\right)} \int_{t_{0}}^{t_{1}}\left[\left(t_{1}-s\right)^{\alpha_{i}-1}-\left(t_{2}-s\right)^{\alpha_{i}-1}\right]\left|f_{i}\left(s, x_{1_{s}}, x_{2_{s}}, \ldots, x_{k_{s}}\right)\right| d s \\
& +\frac{1}{\Gamma\left(\alpha_{i}\right)} \int_{t_{1}}^{t_{2}}\left(t_{2}-s\right)^{\alpha_{i}-1}\left|f_{i}\left(s, x_{1_{s}}, x_{2_{s}}, \ldots, x_{k_{s}}\right)\right| d s \\
\leq & \frac{1}{\Gamma\left(\alpha_{i}\right)}\left\{\int_{t_{0}}^{t_{1}}\left[\left(t_{1}-s\right)^{\alpha_{i}-1}-\left(t_{2}-s\right)^{\alpha_{i}-1}\right]^{\frac{1}{1-\alpha_{i 1}}} d s\right\}^{1-\alpha_{i 1}} \\
& \times\left[\int_{t_{0}}^{t_{1}}\left|f_{i}\left(s, x_{1_{s}}, x_{2_{s}}, \ldots, x_{k_{s}}\right)\right|^{\frac{1}{\alpha_{i 1}}} d s\right]^{\alpha_{i 1}} \\
& +\frac{1}{\Gamma\left(\alpha_{i}\right)}\left[\int_{t_{1}}^{t_{2}}\left(t_{2}-s\right)^{\frac{\alpha_{i}-1}{1-\alpha_{i 1}}} d s\right]^{1-\alpha_{i 1}}\left[\int_{t_{1}}^{t_{2}}\left|f_{i}\left(s, x_{1_{s}}, x_{2_{s}}, \ldots, x_{k_{s}}\right)\right|^{\frac{1}{\alpha_{i 1}}} d s\right]^{\alpha_{i 1}} \\
\leq & \frac{1}{\Gamma\left(\alpha_{i}\right)}\left(\frac{1}{1+v_{i 1}}\right)^{1-\alpha_{i 1}}\left[\left(t_{1}-t_{0}\right)^{\frac{\alpha_{i}-1}{1-\alpha_{i 1}}+1}+\left(t_{2}-t_{1}\right)^{\frac{\alpha_{i}-1}{1-\alpha_{i 1}}+1}-\left(t_{2}-t_{0}\right)^{\frac{\alpha_{i}-1}{1-\alpha_{i 1}}+1}\right]^{1-\alpha_{i 1}} \\
& \times\left[\int_{t_{0}}^{\tilde{T}^{\prime}}\left|f_{i}\left(s, x_{1_{s}}, x_{2_{s}}, \ldots, x_{k_{s}}\right)\right|^{\frac{1}{\alpha_{i 1}}} d s\right]^{\alpha_{i 1}} \\
& +\frac{1}{\Gamma\left(\alpha_{i}\right)}\left(\frac{1}{1+v_{i 1}}\right)^{1-\alpha_{i 1}}\left[\left(t_{2}-t_{1}\right)^{\frac{\alpha_{i}-1}{1-\alpha_{i 1}}+1}\right]^{1-\alpha_{i 1}}\left[\int_{t_{0}}^{\tilde{T}^{\prime}}\left|f_{i}\left(s, x_{1_{s}}, x_{2_{s}}, \ldots, x_{k_{s}}\right)\right|^{\frac{1}{\alpha_{i 1}}} d s\right]^{\alpha_{i 1}} \\
\leq & \frac{1}{\Gamma\left(\alpha_{i}\right)}\left(\frac{1}{1+v_{i 1}}\right)^{1-\alpha_{i 1}}\left[\int_{t_{0}}^{\tilde{T}^{\prime}}\left|f_{i}\left(s, x_{1_{s}}, x_{2_{s}}, \ldots, x_{k_{s}}\right)\right|^{\frac{1}{\alpha_{i 1}}} d s\right]^{\alpha_{i 1}}\left(t_{2}-t_{1}\right)^{\alpha_{i}-\alpha_{i 1}}
\end{aligned}
$$

and so $\lim _{t_{2} \rightarrow t_{1}}\left|T_{i}\left(x_{1}, x_{2}, \ldots, x_{k}\right)\left(t_{2}\right)-T_{i}\left(x_{1}, x_{2}, \ldots, x_{k}\right)\left(t_{1}\right)\right|=0$. If $t_{1}, t_{2}>\tilde{T}^{\prime}$, then

$$
\begin{aligned}
& \left|T_{i}\left(x_{1}, x_{2}, \ldots, x_{k}\right)\left(t_{2}\right)-T_{i}\left(x_{1}, x_{2}, \ldots, x_{k}\right)\left(t_{1}\right)\right| \\
& =\mid \frac{1}{\Gamma\left(\alpha_{i}\right)} \int_{t_{0}}^{t_{2}}\left(t_{2}-s\right)^{\alpha_{i}-1} f_{i}\left(s, x_{1_{s}}, x_{2_{s}}, \ldots, x_{k_{s}}\right) d s \\
& \quad-\frac{1}{\Gamma\left(\alpha_{i}\right)} \int_{t_{0}}^{t_{1}}\left(t_{1}-s\right)^{\alpha_{i}-1} f_{i}\left(s, x_{1_{s}}, x_{2_{s}}, \ldots, x_{k_{s}}\right) d s \mid \\
& \quad \leq\left(t_{2}-t_{0}\right)^{-\gamma_{i 1}}+\left(t_{1}-t_{0}\right)^{-\gamma_{i 1}} \leq \epsilon .
\end{aligned}
$$

Now, let $t_{0}<t_{1}<\tilde{T}^{\prime}<t_{2}$. Since

$$
\begin{aligned}
& \left|T_{i}\left(x_{1}, x_{2}, \ldots, x_{k}\right)\left(t_{2}\right)-T_{i}\left(x_{1}, x_{2}, \ldots, x_{k}\right)\left(t_{1}\right)\right| \\
& \quad \leq\left|T_{i}\left(x_{1}, x_{2}, \ldots, x_{k}\right)\left(t_{2}\right)-T_{i}\left(x_{1}, x_{2}, \ldots, x_{k}\right)\left(\tilde{T}^{\prime}\right)\right| \\
& \quad+\left|T_{i}\left(x_{1}, x_{2}, \ldots, x_{k}\right)\left(\tilde{T}^{\prime}\right)-T_{i}\left(x_{1}, x_{2}, \ldots, x_{k}\right)\left(t_{1}\right)\right|,
\end{aligned}
$$

we get $\lim _{t_{2} \rightarrow t_{1}}\left|T_{i}\left(x_{1}, x_{2}, \ldots, x_{k}\right)\left(t_{2}\right)-T_{i}\left(x_{1}, x_{2}, \ldots, x_{k}\right)\left(t_{1}\right)\right|=0$ in all cases. This implies that the set $T\left(S_{1}\right)$ is equi-continuous. Since $T\left(S_{1}\right) \subset S_{1}$ is uniformly bounded, $T\left(S_{1}\right)$ is relatively compact. Now by using Theorem 2.1, $T$ has a fixed point in $S_{1}$ which is a solution of the problem (2.1). Since $x(t)=\left(x_{1}(t), x_{2}(t), \ldots, x_{k}(t)\right) \in S_{1}, \lim _{t \rightarrow \infty} x(t)=0$. Thus, $x(t)$ is an attractive solution for the problem (2.1). 
Theorem 3.2 Suppose that for each $i \in\{1,2, \ldots, k\}$ there exist $\gamma_{i 2}>0, \alpha_{i 2} \in\left(0, \alpha_{i}\right)$ and $l_{i} \in L^{\frac{1}{\alpha_{i 2}}}\left(J, \mathbb{R}^{+}\right)$such that $\frac{1}{\Gamma\left(\alpha_{i}\right)} \int_{t_{0}}^{t}\left(t_{1}-s\right)^{\alpha_{i}-1} l_{i}(s)\left(s-t_{0}\right)^{-\gamma_{i 2}} d s \leq\left(t-t_{0}\right)^{-\gamma_{i 2}}$ and

$$
\left|\frac{\phi_{i}\left(t_{0}\right)}{\Gamma\left(1-\alpha_{i}\right)}\left(t-t_{0}\right)^{-\alpha_{i}}+f_{i}\left(t, x_{1_{t}}, x_{2_{t}}, \ldots, x_{k_{t}}\right)\right| \leq l_{i}(t)\left\|x_{i_{t}}\right\|
$$

for all $i=1,2, \ldots, k, t \in J$ and $x_{i} \in C\left(\left[t_{0}-r, \infty\right), \mathbb{R}^{n}\right)$. Then the problem (2.1) has at least one attractive solution $\left(x_{1}, x_{2}, \ldots, x_{k}\right)$ such that $x_{i} \in C\left(\left[t_{0}-r, \infty\right), \mathbb{R}^{n}\right)$ for all $i=1,2, \ldots, k$.

Proof It is sufficient we consider the set

$$
\begin{aligned}
S_{2}= & \left\{\left(x_{1}, x_{2}, \ldots, x_{k}\right): x_{i} \in C\left(\left[t_{0}-r, \infty\right), \mathbb{R}^{n}\right),\left\|x_{i t}\right\| \leq\left(t-t_{0}\right)^{-\gamma_{i 2}}\right. \\
& \text { for all } \left.i=1,2, \ldots, k \text { and } t \geq \tilde{t}>t_{0}\right\},
\end{aligned}
$$

where $\tilde{t}$ is a constant. By using a similar techniques and proof in Theorem 3.1, one can show that $T\left(S_{2}\right) \subset S_{2}, T$ is continuous and $T\left(S_{2}\right)$ is relatively compact. Now by using Theorem 2.1, $T$ has a fixed point in $S_{2}$ which is a solution of the problem (2.1). Since $x(t)=\left(x_{1}(t), x_{2}(t), \ldots, x_{k}(t)\right) \in S_{2}, \lim _{t \rightarrow \infty} x(t)=0$. Thus, $x(t)$ is an attractive solution for the problem (2.1).

Theorem 3.3 Suppose that for each $i \in\{1,2, \ldots, k\}$ there exists $\beta_{i 1} \in\left(\alpha_{i}, 1\right)$ such that

$$
\left|\frac{\phi_{i}\left(t_{0}\right)}{\Gamma\left(1-\alpha_{i}\right)}\left(t-t_{0}\right)^{-\alpha_{i}}+f_{i}\left(t, x_{1_{t}}, x_{2 t}, \ldots, x_{k_{t}}\right)\right| \leq \frac{\Gamma\left(1+\alpha_{i}-\beta_{i 1}\right)}{\Gamma\left(1-\beta_{i 1}\right)}\left(t-t_{0}\right)^{-\beta_{i 1}}
$$

for all $t \in J$. Then the problem (2.1) has at least one attractive solution $\left(x_{1}, x_{2}, \ldots, x_{k}\right)$ such that $x_{i} \in C\left(\left[t_{0}-r, \infty\right), \mathbb{R}^{n}\right)$ for all $i=1,2, \ldots, k$.

Proof It is sufficient we consider the set

$$
\begin{aligned}
S_{3}= & \left\{\left(x_{1}, x_{2}, \ldots, x_{k}\right): x_{i} \in C\left(\left[t_{0}-r, \infty\right), \mathbb{R}^{n}\right),\left|x_{i}(t)\right| \leq\left(t-t_{0}\right)^{\beta_{i 1}-\alpha_{i}}\right. \\
& \text { for all } \left.i=1,2, \ldots, k \text { and } t \geq \tilde{t}>t_{0}\right\},
\end{aligned}
$$

where $\tilde{t}$ is a constant. By using a similar techniques and proof in Theorem 3.1, one can show that $T\left(S_{3}\right) \subset S_{3}, T$ is continuous and $T\left(S_{3}\right)$ is relatively compact. Now, by using Theorem 2.1, $T$ has a fixed point in $S_{3}$ which is a solution of the problem (2.1). Since $x(t)=\left(x_{1}(t), x_{2}(t), \ldots, x_{k}(t)\right) \in S_{3}, \lim _{t \rightarrow \infty} x(t)=0$. Thus, $x(t)$ is an attractive solution for the problem (2.1).

Here, we are going to investigate global attractivity of solutions of the problem (2.2). We assume that $g_{i}\left(t, x_{1}(t), x_{2}(t), \ldots, x_{k}(t)\right)$ is Lebesgue measurable with respect to $t$ on $\left[t_{0}, \infty\right)$ and there exists a constant $\alpha_{i 1}^{\prime} \in\left(0, \alpha_{i}\right)$ such that $g_{i} \in L^{\frac{1}{\alpha_{i 1}^{\prime}}}\left(J \times \mathbb{R}^{n} \times \mathbb{R}^{n} \times \cdots \times \mathbb{R}^{n}, \mathbb{R}^{n}\right)$ and $g_{i}\left(t, x_{1}(t), x_{2}(t), \ldots, x_{k}(t)\right)$ is continuous with respect to $x_{j}$ on $\left[t_{0}, \infty\right)$ for all $i, j=1,2, \ldots, k$. Note that the problem (2.2) is equivalent to the system of equations

$$
x_{i}(t)=\frac{x_{i}^{0}}{\Gamma\left(\alpha_{i}\right)}\left(t-t_{0}\right)^{\alpha_{i}-1}+\frac{1}{\Gamma\left(\alpha_{i}\right)} \int_{t_{0}}^{t}(t-s)^{\alpha_{i}-1} g_{i}\left(s, x_{1}(s), x_{2}(s), \ldots, x_{k}(s)\right) d s
$$


for all $t>t_{0}$ and $i=1,2, \ldots, k$. Define the operator $T: X^{k} \rightarrow X^{k}$ by

$$
T\left(x_{1}, x_{2}, \ldots, x_{k}\right)(t)=\left(\begin{array}{l}
T_{1}\left(x_{1}, x_{2}, \ldots, x_{k}\right)(t) \\
T_{2}\left(x_{1}, x_{2}, \ldots, x_{k}\right)(t) \\
\vdots \\
T_{k}\left(x_{1}, x_{2}, \ldots, x_{k}\right)(t)
\end{array}\right)
$$

where

$$
T_{i}\left(x_{1}, x_{2}, \ldots, x_{k}\right)(t)=\frac{x_{i}^{0}}{\Gamma\left(\alpha_{i}\right)}\left(t-t_{0}\right)^{\alpha_{i}-1}+\frac{1}{\Gamma\left(\alpha_{i}\right)} \int_{t_{0}}^{t}(t-s)^{\alpha_{i}-1} g_{i}\left(s, x_{1}(s), x_{2}(s), \ldots, x_{k}(s)\right) d s
$$

for all $i=1,2, \ldots, k$. Now, define

$$
A\left(x_{1}, x_{2}, \ldots, x_{k}\right)(t)=\left(\begin{array}{l}
A_{1}\left(x_{1}, x_{2}, \ldots, x_{k}\right)(t) \\
A_{2}\left(x_{1}, x_{2}, \ldots, x_{k}\right)(t) \\
\vdots \\
A_{k}\left(x_{1}, x_{2}, \ldots, x_{k}\right)(t)
\end{array}\right),
$$

where $A_{i}\left(x_{1}, x_{2}, \ldots, x_{k}\right)(t)=\frac{x_{i}^{0}}{\Gamma\left(\alpha_{i}\right)}\left(t-t_{0}\right)^{\alpha_{i}-1}$ for all $i=1,2, \ldots, k$. Finally, define

$$
B\left(x_{1}, x_{2}, \ldots, x_{k}\right)(t)=\left(\begin{array}{l}
B_{1}\left(x_{1}, x_{2}, \ldots, x_{k}\right)(t) \\
B_{2}\left(x_{1}, x_{2}, \ldots, x_{k}\right)(t) \\
\vdots \\
B_{k}\left(x_{1}, x_{2}, \ldots, x_{k}\right)(t)
\end{array}\right),
$$

where $B_{i}\left(x_{1}, x_{2}, \ldots, x_{k}\right)(t)=\frac{1}{\Gamma\left(\alpha_{i}\right)} \int_{t_{0}}^{t}(t-s)^{\alpha_{i}-1} g_{i}\left(s, x_{1}(s), x_{2}(s), \ldots, x_{k}(s)\right) d s$ for all $i=1,2, \ldots, k$. It is easy to check that $\left(x_{1}(t), x_{2}(t), \ldots, x_{k}(t)\right)$ is a solution of the problem (2.2) if and only if it is a fixed point of the operator $T$. Note that $A$ is a contraction with constant 0 .

Theorem 3.4 Suppose that for each $i \in\{1,2, \ldots, k\}$ there exist $\alpha_{i}<\beta_{i 1}^{\prime}<1$ and $M_{i} \geq 0$ such that $\left|g_{i}\left(t, x_{1}(t), x_{2}(t), \ldots, x_{k}(t)\right)\right| \leq M_{i}\left(t-t_{0}\right)^{-\beta_{i 1}^{\prime}}$ for all $t \in J$ and $x_{1}, \ldots, x_{k} \in C\left(\left(t_{0}, \infty\right), \mathbb{R}^{n}\right)$. Then the zero solution of the problem (2.2) is globally attractive.

Proof Consider the set

$$
\begin{aligned}
S_{1}^{\prime}= & \left\{\left(x_{1}, x_{2}, \ldots, x_{k}\right): x_{i} \in C\left(\left(t_{0}, \infty\right), \mathbb{R}^{n}\right),\left|x_{i}(t)\right| \leq\left(t-t_{0}\right)^{-\gamma_{i 1}^{\prime}}\right. \\
& \text { for all } \left.i=1,2, \ldots, k \text { and } t \geq t_{0}+\tilde{T}_{1}\right\}
\end{aligned}
$$

where $\gamma_{i 1}^{\prime}=\frac{1}{2}\left(\beta_{i 1}^{\prime}-\alpha_{i}\right)$ and $\tilde{T}_{1}$ is chosen such that $\frac{\left|x_{i}^{0}\right|}{\Gamma\left(\alpha_{i}\right)} \tilde{T}_{1}^{\frac{1}{2}\left(\alpha_{i}-1\right)}+\frac{M_{i} \Gamma\left(1-\beta_{i 1}^{\prime}\right)}{\Gamma\left(1+\alpha_{i}-\beta_{i 1}^{\prime}\right)} \tilde{T}_{1}^{-\frac{1}{2}\left(\beta_{i 1}^{\prime}-\alpha_{i}\right)} \leq 1$ for all $i=1,2, \ldots, k$. First, we show that $B$ maps $S_{1}^{\prime}$ into $S_{1}^{\prime}$. It is easy to check that $S_{1}^{\prime}$ is a closed, bounded, and convex subset of $\mathbb{R}^{n} \times \mathbb{R}^{n} \times \cdots \times \mathbb{R}^{n}$. Note that

$$
\begin{aligned}
& \left|B_{i}\left(y_{1}, y_{2}, \ldots, y_{k}\right)(t)\right| \\
& \quad \leq \frac{1}{\Gamma\left(\alpha_{i}\right)} \int_{t_{0}}^{t}(t-s)^{\alpha_{i}-1}\left|g_{i}\left(s, x_{1}(s), x_{2}(s), \ldots, x_{k}(s)\right)\right| d s
\end{aligned}
$$




$$
\begin{aligned}
& \leq \frac{1}{\Gamma\left(\alpha_{i}\right)} \int_{t_{0}}^{t}(t-s)^{\alpha_{i}-1} M_{i}\left(s-t_{0}\right)^{-\beta_{i 1}^{\prime}} d s \\
& \leq \frac{M_{i} \Gamma\left(1-\beta_{i 1}^{\prime}\right)}{\Gamma\left(1+\alpha_{i}-\beta_{i 1}^{\prime}\right)}\left(t-t_{0}\right)^{-\left(\beta_{i 1}^{\prime}-\alpha_{i}\right)}
\end{aligned}
$$

and $\frac{M_{i} \Gamma\left(1-\beta_{i 1}^{\prime}\right)}{\Gamma\left(1+\alpha_{i}-\beta_{i 1}^{\prime}\right)}\left(t-t_{0}\right)^{-\frac{1}{2}\left(\beta_{i 1}^{\prime}-\alpha_{i}\right)} \leq \frac{M_{i} \Gamma\left(1-\beta_{11}^{\prime}\right)}{\Gamma\left(1+\alpha_{i}-\beta_{i 1}^{\prime}\right)} \tilde{T}_{1}^{-\frac{1}{2}\left(\beta_{i 1}^{\prime}-\alpha_{i}\right)} \leq 1$ for all $i=1,2, \ldots, k$ and $t \geq t_{0}+\tilde{T}_{1}$. Thus,

$$
\left|B_{i}\left(y_{1}, y_{2}, \ldots, y_{k}\right)(t)\right| \leq\left[\frac{M_{i} \Gamma\left(1-\beta_{i 1}^{\prime}\right)}{\Gamma\left(1+\alpha_{i}-\beta_{i 1}^{\prime}\right)}\left(t-t_{0}\right)^{-\frac{1}{2}\left(\beta_{i 1}^{\prime}-\alpha_{i}\right)}\right]\left(t-t_{0}\right)^{-\frac{1}{2}\left(\beta_{i 1}^{\prime}-\alpha_{i}\right)} \leq\left(t-t_{0}\right)^{-\gamma_{i 1}^{\prime}}
$$

for all $i=1,2, \ldots, k$ and $t \geq t_{0}+\tilde{T}_{1}$. Hence, $B\left(S_{1}^{\prime}\right) \subset S_{1}^{\prime}$. Now, we show that $B$ is continuous on $\left[t_{0}+\tilde{T}_{1}, \infty\right)$. Let $\left(y_{1}^{m}, y_{2}^{m}, \ldots, y_{k}^{m}\right),\left(y_{1}, y_{2}, \ldots, y_{k}\right) \in S_{1}^{\prime}$ for all $m \geq 1$ and $\lim _{m \rightarrow \infty} \mid y_{i}^{m}(t)-$ $y_{i}(t) \mid=0$. Then, one can get $\lim _{m \rightarrow \infty} g_{i}\left(t, y_{1}^{m}(t), y_{2}^{m}(t), \ldots, y_{k}^{m}(t)\right)=g_{i}\left(t, y_{1}(t), y_{2}(t), \ldots, y_{k}(t)\right)$ for all $t \geq t_{0}+\tilde{T}_{1}$. Let $\epsilon>0$ be given. Choose $\tilde{T}>t_{0}+\tilde{T}_{1}$ such that $\frac{M_{i} \Gamma\left(1-\beta_{1}^{\prime}\right)}{\Gamma\left(1+\alpha_{i}-\beta_{i 1}^{\prime}\right)}\left(\tilde{T}-t_{0}\right)^{-\left(\beta_{i 1}^{\prime}-\alpha_{i}\right)}<$ $\frac{\epsilon}{2}$ for all $t>\tilde{T}$. Let $v_{i 1}^{\prime}=\frac{\alpha_{i}-1}{1-\alpha_{i 1}^{\prime}}$ for $i=1,2, \ldots, k$. Then, we have

$$
\begin{aligned}
\left|B_{i}\left(y_{1}^{m}, y_{2}^{m}, \ldots, y_{k}^{m}\right)(t)-B_{i}\left(y_{1}, y_{2}, \ldots, y_{k}\right)(t)\right| \\
\leq \frac{1}{\Gamma\left(\alpha_{i}\right)} \int_{t_{0}}^{t}(t-s)^{\alpha_{i}-1}\left|g_{i}\left(s, y_{1}^{m}(s), y_{2}^{m}(s), \ldots, y_{k}^{m}(s)\right)-g_{i}\left(s, y_{1}(s), y_{2}(s), \ldots, y_{k}(s)\right)\right| d s \\
\leq \frac{1}{\Gamma\left(\alpha_{i}\right)}\left\{\int_{t_{0}}^{t}\left[(t-s)^{\alpha_{i}-1}\right]^{\frac{1}{1-\alpha_{i 1}^{\prime}}} d s\right\}^{1-\alpha_{i 1}^{\prime}} \\
\quad \times\left[\int_{t_{0}}^{t}\left|g_{i}\left(s, y_{1}^{m}(s), y_{2}^{m}(s), \ldots, y_{k}^{m}(s)\right)-g_{i}\left(s, y_{1}(s), y_{2}(s), \ldots, y_{k}(s)\right)\right|^{\frac{1}{\alpha_{i 1}^{\prime}}} d s\right]^{\alpha_{i 1}^{\prime}} \\
\leq \frac{1}{\Gamma\left(\alpha_{i}\right)}\left(\frac{1}{1+v_{i 1}^{\prime}}\left(t-t_{0}\right)^{1+v_{i 1}^{\prime}}\right)^{1-\alpha_{i 1}^{\prime}} \\
\quad \times\left[\int_{t_{0}}^{\tilde{T}}\left|g_{i}\left(s, y_{1}^{m}(s), y_{2}^{m}(s), \ldots, y_{k}^{m}(s)\right)-g_{i}\left(s, y_{1}(s), y_{2}(s), \ldots, y_{k}(s)\right)\right|^{\frac{1}{\alpha_{i 1}^{\prime}}} d s\right]^{\alpha_{i 1}^{\prime}} \\
\leq \frac{1}{\Gamma\left(\alpha_{i}\right)}\left(\frac{1}{1+v_{i 1}^{\prime}}\left(\tilde{T}-t_{0}\right)^{1+v_{i 1}^{\prime}}\right)^{1-\alpha_{i 1}^{\prime}}\left(\tilde{T}-t_{0}\right)^{\alpha_{i 1}^{\prime}} \\
\quad \times \sup _{t_{0} \leq s \leq \tilde{T}}\left|g_{i}\left(s, y_{1}^{m}(s), y_{2}^{m}(s), \ldots, y_{k}^{m}(s)\right)-g_{i}\left(s, y_{1}(s), y_{2}(s), \ldots, y_{k}(s)\right)\right|
\end{aligned}
$$

for all $t_{0}+\tilde{T}_{1} \leq t \leq \tilde{T}$. Hence, $\lim _{m \rightarrow \infty}\left|B_{i}\left(y_{1}^{m}, y_{2}^{m}, \ldots, y_{k}^{m}\right)(t)-B_{i}\left(y_{1}, y_{2}, \ldots, y_{k}\right)(t)\right|=0$ for all $t_{0}+\tilde{T}_{1} \leq t \leq \tilde{T}$. Also,

$$
\begin{aligned}
& \left|B_{i}\left(y_{1}^{m}, y_{2}^{m}, \ldots, y_{k}^{m}\right)(t)-B_{i}\left(y_{1}, y_{2}, \ldots, y_{k}\right)(t)\right| \\
& \quad \leq \frac{1}{\Gamma\left(\alpha_{i}\right)} \int_{t_{0}}^{t}(t-s)^{\alpha_{i}-1}\left|g_{i}\left(s, y_{1}^{m}(s), y_{2}^{m}(s), \ldots, y_{k}^{m}(s)\right)-g_{i}\left(s, y_{1}(s), y_{2}(s), \ldots, y_{k}(s)\right)\right| d s \\
& \quad \leq \frac{1}{\Gamma\left(\alpha_{i}\right)} \int_{t_{0}}^{t}(t-s)^{\alpha_{i}-1}\left[\left|g_{i}\left(s, y_{1}^{m}(s), y_{2}^{m}(s), \ldots, y_{k}^{m}(s)\right)\right|+\left|g_{i}\left(s, y_{1}(s), y_{2}(s), \ldots, y_{k}(s)\right)\right|\right] d s \\
& \quad \leq \frac{1}{\Gamma\left(\alpha_{i}\right)} \int_{t_{0}}^{t}(t-s)^{\alpha_{i}-1}\left[2 M_{i}\left(s-t_{0}\right)^{-\beta_{i 1}^{\prime}}\right] d s
\end{aligned}
$$




$$
\begin{aligned}
& \leq \frac{2 M_{i} \Gamma\left(1-\beta_{i 1}^{\prime}\right)}{\Gamma\left(1+\alpha_{i}-\beta_{i 1}^{\prime}\right)}\left(t-t_{0}\right)^{-\left(\beta_{i 1}^{\prime}-\alpha_{i}\right)} \\
& \leq \frac{2 M_{i} \Gamma\left(1-\beta_{i 1}^{\prime}\right)}{\Gamma\left(1+\alpha_{i}-\beta_{i 1}^{\prime}\right)}\left(\tilde{T}-t_{0}\right)^{-\left(\beta_{i 1}^{\prime}-\alpha_{i}\right)} \leq \epsilon
\end{aligned}
$$

for all $t>\tilde{T}$. Thus, $\lim _{m \rightarrow \infty}\left|B_{i}\left(y_{1}^{m}, y_{2}^{m}, \ldots, y_{k}^{m}\right)(t)-B_{i}\left(y_{1}, y_{2}, \ldots, y_{k}\right)(t)\right|=0$ for all $t \geq t_{0}+\tilde{T}_{1}$. This implies that $B_{i}$ is continuous on $\left[t_{0}+\tilde{T}_{1}, \infty\right)$ for $i=1,2, \ldots, k$ and so $B$ is continuous on $\left[t_{0}+\tilde{T}_{1}, \infty\right)$. Now, we show that $B\left(S_{1}^{\prime}\right)$ is equi-continuous. Let $\epsilon>0$ be given. Since $\lim _{t \rightarrow \infty}\left(t-t_{0}\right)^{-\gamma_{i 1}^{\prime}}=0$, there exists $\tilde{T}^{\prime}>t_{0}+\tilde{T}_{1}$ such that $\left(t-t_{0}\right)^{-\gamma_{i 1}^{\prime}}<\frac{\epsilon}{2}$ for $t>\tilde{T}^{\prime}$. Let $t_{1}, t_{2} \geq t_{0}+\tilde{T}_{1}$ and $t_{2}>t_{1}$. If $t_{1}, t_{2} \in\left[t_{0}+\tilde{T}_{1}, \tilde{T}^{\prime}\right]$, then we have

$$
\begin{aligned}
& \left|B_{i}\left(y_{1}, y_{2}, \ldots, y_{k}\right)\left(t_{2}\right)-B_{i}\left(y_{1}, y_{2}, \ldots, y_{k}\right)\left(t_{1}\right)\right| \\
& =\mid \frac{1}{\Gamma\left(\alpha_{i}\right)} \int_{t_{0}}^{t_{2}}\left(t_{2}-s\right)^{\alpha_{i}-1} g_{i}\left(s, y_{1}(s), y_{2}(s), \ldots, y_{k}(s)\right) d s \\
& -\frac{1}{\Gamma\left(\alpha_{i}\right)} \int_{t_{0}}^{t_{1}}\left(t_{1}-s\right)^{\alpha_{i}-1} g_{i}\left(s, y_{1}(s), y_{2}(s), \ldots, y_{k}(s)\right) d s \mid \\
& \leq \frac{1}{\Gamma\left(\alpha_{i}\right)} \int_{t_{0}}^{t_{1}}\left[\left(t_{1}-s\right)^{\alpha_{i}-1}-\left(t_{2}-s\right)^{\alpha_{i}-1}\right]\left|g_{i}\left(s, y_{1}(s), y_{2}(s), \ldots, y_{k}(s)\right)\right| d s \\
& +\frac{1}{\Gamma\left(\alpha_{i}\right)} \int_{t_{1}}^{t_{2}}\left(t_{2}-s\right)^{\alpha_{i}-1}\left|g_{i}\left(s, y_{1}(s), y_{2}(s), \ldots, y_{k}(s)\right)\right| d s \\
& \leq \frac{1}{\Gamma\left(\alpha_{i}\right)}\left[\int_{t_{0}}^{t_{1}}\left[\left(t_{1}-s\right)^{\alpha_{i}-1}-\left(t_{2}-s\right)^{\alpha_{i}-1}\right]^{\frac{1}{1-\alpha_{i 1}^{\prime}}} d s\right]^{1-\alpha_{i 1}^{\prime}} \\
& \times\left[\int_{t_{0}}^{t_{1}}\left|g_{i}\left(s, y_{1}(s), y_{2}(s), \ldots, y_{k}(s)\right)\right|^{\frac{1}{\alpha_{i 1}^{\prime}}} d s\right]^{\alpha_{i 1}^{\prime}} \\
& +\frac{1}{\Gamma\left(\alpha_{i}\right)}\left[\int_{t_{1}}^{t_{2}}\left(t_{2}-s\right)^{\frac{\alpha_{i}-1}{1-\alpha_{i 1}^{\prime}}} d s\right]^{1-\alpha_{i 1}^{\prime}}\left[\int_{t_{0}}^{t_{1}}\left|g_{i}\left(s, y_{1}(s), y_{2}(s), \ldots, y_{k}(s)\right)\right|^{\frac{1}{\alpha_{i 1}^{\prime}}} d s\right]^{\alpha_{i 1}^{\prime}} \\
& \leq \frac{1}{\Gamma\left(\alpha_{i}\right)}\left(\frac{1}{1+v_{i 1}^{\prime}}\right)^{1-\alpha_{i 1}^{\prime}}\left(\left(t_{1}-t_{0}\right)^{1+v_{i 1}^{\prime}}-\left(t_{2}-t_{0}\right)^{1+v_{i 1}^{\prime}}+\left(t_{2}-t_{1}\right)^{1+v_{i 1}^{\prime}}\right)^{1-\alpha_{i 1}^{\prime}} \\
& \times\left[\int_{t_{0}}^{\tilde{T}^{\prime}}\left|g_{i}\left(s, y_{1}(s), y_{2}(s), \ldots, y_{k}(s)\right)\right|^{\frac{1}{\alpha_{i 1}^{\prime}}} d s\right]^{\alpha_{i 1}^{\prime}} \\
& +\frac{1}{\Gamma\left(\alpha_{i}\right)}\left(\frac{1}{1+v_{i 1}^{\prime}}\right)^{1-\alpha_{i 1}^{\prime}}\left(\left(t_{2}-t_{1}\right)^{1+v_{i 1}^{\prime}}\right)^{1-\alpha_{i 1}^{\prime}}\left[\int_{t_{0}}^{\tilde{T}^{\prime}}\left|g_{i}\left(s, y_{1}(s), y_{2}(s), \ldots, y_{k}(s)\right)\right|^{\frac{1}{\alpha_{i 1}^{\prime}}} d s\right]^{\alpha_{i 1}^{\prime}} \\
& \leq \frac{2}{\Gamma\left(\alpha_{i}\right)}\left(\frac{1}{1+v_{i 1}^{\prime}}\right)^{1-\alpha_{i 1}^{\prime}}\left[\int_{t_{0}}^{\tilde{T}^{\prime}}\left|g_{i}\left(s, y_{1}(s), y_{2}(s), \ldots, y_{k}(s)\right)\right|^{\frac{1}{\alpha_{i 1}^{\prime}}} d s\right]^{\alpha_{i 1}^{\prime}}\left(t_{2}-t_{1}\right)^{\alpha_{i}-\alpha_{i 1}^{\prime}}
\end{aligned}
$$

and so $\lim _{t_{2} \rightarrow t_{1}}\left|B_{i}\left(y_{1}, y_{2}, \ldots, y_{k}\right)\left(t_{2}\right)-B_{i}\left(y_{1}, y_{2}, \ldots, y_{k}\right)\left(t_{1}\right)\right|=0$. If $t_{1}, t_{2}>\tilde{T}^{\prime}$, then

$$
\begin{aligned}
& \left|B_{i}\left(y_{1}, y_{2}, \ldots, y_{k}\right)\left(t_{2}\right)-B_{i}\left(y_{1}, y_{2}, \ldots, y_{k}\right)\left(t_{1}\right)\right| \\
& \leq \frac{1}{\Gamma\left(\alpha_{i}\right)} \int_{t_{0}}^{t_{2}}\left(t_{2}-s\right)^{\alpha_{i}-1}\left|g_{i}\left(s, y_{1}(s), y_{2}(s), \ldots, y_{k}(s)\right)\right| d s \\
& \quad+\frac{1}{\Gamma\left(\alpha_{i}\right)} \int_{t_{0}}^{t_{1}}\left(t_{1}-s\right)^{\alpha_{i}-1}\left|g_{i}\left(s, y_{1}(s), y_{2}(s), \ldots, y_{k}(s)\right)\right| d s \\
& \leq\left(t_{2}-t_{0}\right)^{-\gamma_{i 1}^{\prime}}+\left(t_{1}-t_{0}\right)^{-\gamma_{i 1}^{\prime}} \leq \epsilon .
\end{aligned}
$$


If $t_{0}+\tilde{T}_{1} \leq t_{1}<\tilde{T}^{\prime}<t_{2}$, then we have

$$
\begin{aligned}
& \left|B_{i}\left(y_{1}, y_{2}, \ldots, y_{k}\right)\left(t_{2}\right)-B_{i}\left(y_{1}, y_{2}, \ldots, y_{k}\right)\left(t_{1}\right)\right| \\
& \leq\left|B_{i}\left(y_{1}, y_{2}, \ldots, y_{k}\right)\left(t_{2}\right)-B_{i}\left(y_{1}, y_{2}, \ldots, y_{k}\right)\left(\tilde{T}^{\prime}\right)\right| \\
& \quad+\left|B_{i}\left(y_{1}, y_{2}, \ldots, y_{k}\right)\left(\tilde{T}^{\prime}\right)-B_{i}\left(y_{1}, y_{2}, \ldots, y_{k}\right)\left(t_{1}\right)\right|
\end{aligned}
$$

and so $\lim _{t_{2} \rightarrow t_{1}}\left|B_{i}\left(y_{1}, y_{2}, \ldots, y_{k}\right)\left(t_{2}\right)-B_{i}\left(y_{1}, y_{2}, \ldots, y_{k}\right)\left(t_{1}\right)\right|=0$. Thus, $B\left(S_{1}^{\prime}\right)$ is equi-continuous. Since $B\left(S_{1}^{\prime}\right) \subset S_{1}^{\prime}$ is uniformly bounded, $B\left(S_{1}^{\prime}\right)$ is relatively compact. Now, suppose that $x=\left(x_{1}, x_{2}, \ldots, x_{k}\right) \in C\left(\left(t_{0}, \infty\right), \mathbb{R}^{n}\right) \times C\left(\left(t_{0}, \infty\right), \mathbb{R}^{n}\right) \times \cdots \times C\left(\left(t_{0}, \infty\right), \mathbb{R}^{n}\right), y=$ $\left(y_{1}, y_{2}, \ldots, y_{k}\right) \in S_{1}^{\prime}$ and $x=A x+B y$. Then,

$$
\begin{aligned}
\left|x_{i}(t)\right| & \leq\left|A_{i}\left(x_{1}, x_{2}, \ldots, x_{k}\right)(t)\right|+\left|B_{i}\left(y_{1}, y_{2}, \ldots, y_{k}\right)(t)\right| \\
& \leq \frac{\left|x_{i}^{0}\right|}{\Gamma\left(\alpha_{i}\right)}\left(t-t_{0}\right)^{\alpha_{i}-1}+\frac{1}{\Gamma\left(\alpha_{i}\right)} \int_{t_{0}}^{t}(t-s)^{\alpha_{i}-1}\left|g_{i}\left(s, y_{1}(s), y_{2}(s), \ldots, y_{k}(s)\right)\right| d s \\
& \leq \frac{\left|x_{i}^{0}\right|}{\Gamma\left(\alpha_{i}\right)}\left(t-t_{0}\right)^{\alpha_{i}-1}+\frac{M_{i} \Gamma\left(1-\beta_{i 1}^{\prime}\right)}{\Gamma\left(1+\alpha_{i}-\beta_{i 1}^{\prime}\right)}\left(t-t_{0}\right)^{-\left(\beta_{i 1}^{\prime}-\alpha_{i}\right)}
\end{aligned}
$$

for all $i=1,2, \ldots, k$. Since $0<\alpha_{i}<\beta_{i 1}^{\prime}<1$ for $i=1,2, \ldots, k$, we get

$$
\begin{aligned}
& \frac{\left|x_{i}^{0}\right|}{\Gamma\left(\alpha_{i}\right)}\left(t-t_{0}\right)^{\frac{1}{2}\left(\alpha_{i}-1\right)}+\frac{M_{i} \Gamma\left(1-\beta_{i 1}^{\prime}\right)}{\Gamma\left(1+\alpha_{i}-\beta_{i 1}^{\prime}\right)}\left(t-t_{0}\right)^{-\frac{1}{2}\left(\beta_{i 1}^{\prime}-\alpha_{i}\right)} \\
& \quad \leq \frac{\left|x_{i}^{0}\right|}{\Gamma\left(\alpha_{i}\right)} \tilde{T}_{1}^{\frac{1}{2}\left(\alpha_{i}-1\right)}+\frac{M_{i} \Gamma\left(1-\beta_{i 1}^{\prime}\right)}{\Gamma\left(1+\alpha_{i}-\beta_{i 1}^{\prime}\right)} \tilde{T}_{1}^{-\frac{1}{2}\left(\beta_{i 1}^{\prime}-\alpha_{i}\right)} \leq 1
\end{aligned}
$$

Thus, $\left|x_{i}(t)\right| \leq\left[\frac{\left|x_{i}^{0}\right|}{\Gamma\left(\alpha_{i}\right)}\left(t-t_{0}\right)^{\frac{1}{2}\left(\alpha_{i}-1\right)}+\frac{M_{i} \Gamma\left(1-\beta_{i 1}^{\prime}\right)}{\Gamma\left(1+\alpha_{i}-\beta_{i 1}^{\prime}\right)}\left(t-t_{0}\right)^{-\frac{1}{2}\left(\beta_{i 1}^{\prime}-\alpha_{i}\right)}\right]\left(t-t_{0}\right)^{-\gamma_{i 1}^{\prime}} \leq\left(t-t_{0}\right)^{-\gamma_{i 1}^{\prime}}$ for all $t \geq t_{0}+\tilde{T}_{1}$ and $i=1,2, \ldots, k$. This implies that $x(t)=\left(x_{1}(t), x_{2}(t), \ldots, x_{k}(t)\right) \in S_{1}^{\prime}$ for all $t \geq t_{0}+\tilde{T}_{1}$. Therefore, by using Theorem 2.2 $T$ has a fixed point in $S_{1}^{\prime}$ which is a solution of the problem (2.2). Since all elements of the set $S_{1}^{\prime}$ tend to 0 as $t \rightarrow \infty$, the zero solution of the problem (2.2) is globally attractive.

Theorem 3.5 Suppose that for each $i \in\{1,2, \ldots, k\}$ there exist $\alpha_{i}<\beta_{i 2}^{\prime}<\frac{1}{2}\left(1+\alpha_{i}\right)$ and $l_{i} \geq 0$ such that $\left|g_{i}\left(t, x_{1}(t), x_{2}(t), \ldots, x_{k}(t)\right)\right| \leq l_{i}\left(t-t_{0}\right)^{-\beta_{i 2}^{\prime}}\left|x_{i}(t)\right|$ for all $t \in J$ and $x_{1}, \ldots, x_{k} \in$ $C\left(\left(t_{0}, \infty\right), \mathbb{R}^{n}\right)$. Then the zero solution of the problem (2.2) is globally attractive.

Proof It is sufficient to consider the set

$$
\begin{aligned}
S_{2}^{\prime}= & \left\{\left(x_{1}, x_{2}, \ldots, x_{k}\right): x_{i} \in C\left(\left(t_{0}, \infty\right), \mathbb{R}^{n}\right),\left|x_{i}(t)\right| \leq\left(t-t_{0}\right)^{-\gamma_{i 2}^{\prime}}\right. \\
& \text { for all } \left.i=1,2, \ldots, k \text { and } t \geq t_{0}+\tilde{T}_{2}\right\}
\end{aligned}
$$

where $\gamma_{i 2}^{\prime}=\frac{1}{2}\left(1-\alpha_{i}\right)$ and $\tilde{T}_{2}$ is chosen such that $\frac{\left|x_{i}^{0}\right|}{\Gamma\left(\alpha_{i}\right)} \tilde{T}_{2}^{\frac{1}{2}\left(\alpha_{i}-1\right)}+\frac{l_{i} \Gamma\left(1-\beta_{i 2}^{\prime}-\gamma_{i 2}^{\prime}\right)}{\Gamma\left(1+\alpha_{i}-\beta_{i 2}^{\prime}-\gamma_{i 2}^{\prime}\right)} \tilde{T}_{2}^{-\left(\beta_{i 2}^{\prime}-\alpha_{i}\right)} \leq 1$ for all $i=1,2, \ldots, k$. Similar to the proof of Theorem 3.4, one can show that $S_{2}^{\prime}$ is a closed, bounded, and convex set, $B$ maps $S_{2}^{\prime}$ into $S_{2}^{\prime}, B\left(S_{2}^{\prime}\right)$ is relatively compact, and $B$ is continuous on $\left[t_{0}+\tilde{T}_{2}, \infty\right)$. Now, suppose that $x=\left(x_{1}, x_{2}, \ldots, x_{k}\right) \in C\left(\left(t_{0}, \infty\right), \mathbb{R}^{n}\right) \times C\left(\left(t_{0}, \infty\right), \mathbb{R}^{n}\right) \times$ 
$\cdots \times C\left(\left(t_{0}, \infty\right), \mathbb{R}^{n}\right), y=\left(y_{1}, y_{2}, \ldots, y_{k}\right) \in S_{2}$ and $x=A x+B y$. Then,

$$
\begin{aligned}
\left|x_{i}(t)\right| & \leq\left|A_{i}\left(x_{1}, x_{2}, \ldots, x_{k}\right)(t)\right|+\left|B_{i}\left(y_{1}, y_{2}, \ldots, y_{k}\right)(t)\right| \\
& \leq \frac{\left|x_{i}^{0}\right|}{\Gamma\left(\alpha_{i}\right)}\left(t-t_{0}\right)^{\alpha_{i}-1}+\frac{1}{\Gamma\left(\alpha_{i}\right)} \int_{t_{0}}^{t}(t-s)^{\alpha_{i}-1}\left|g_{i}\left(s, y_{1}(s), y_{2}(s), \ldots, y_{k}(s)\right)\right| d s \\
& \leq \frac{\left|x_{i}^{0}\right|}{\Gamma\left(\alpha_{i}\right)}\left(t-t_{0}\right)^{\alpha_{i}-1}+\frac{1}{\Gamma\left(\alpha_{i}\right)} \int_{t_{0}}^{t}(t-s)^{\alpha_{i}-1} l_{i}\left(s-t_{0}\right)^{-\beta_{i 2}^{\prime}}\left|y_{i}(s)\right| d s \\
& \leq \frac{\left|x_{i}^{0}\right|}{\Gamma\left(\alpha_{i}\right)}\left(t-t_{0}\right)^{\alpha_{i}-1}+\frac{1}{\Gamma\left(\alpha_{i}\right)} \int_{t_{0}}^{t}(t-s)^{\alpha_{i}-1} l_{i}\left(s-t_{0}\right)^{-\beta_{i 2}^{\prime}-\gamma_{i 2}^{\prime}} d s \\
& \leq \frac{\left|x_{i}^{0}\right|}{\Gamma\left(\alpha_{i}\right)}\left(t-t_{0}\right)^{\alpha_{i}-1}+\frac{l_{i} \Gamma\left(1-\beta_{i 2}^{\prime}-\gamma_{i 2}^{\prime}\right)}{\Gamma\left(1+\alpha_{i}-\beta_{i 2}^{\prime}-\gamma_{i 2}^{\prime}\right)}\left(t-t_{0}\right)^{-\left(\beta_{i 2}^{\prime}-\gamma_{i 2}^{\prime}-\alpha_{i}\right)}
\end{aligned}
$$

for all $i=1,2, \ldots, k$. Since $0<\alpha_{i}<\beta_{i 2}^{\prime}<\frac{1}{2}\left(1+\alpha_{i}\right)<1$ for $i=1,2, \ldots, k$, we get

$$
\begin{aligned}
& \frac{\left|x_{i}^{0}\right|}{\Gamma\left(\alpha_{i}\right)}\left(t-t_{0}\right)^{\frac{1}{2}\left(\alpha_{i}-1\right)}+\frac{l_{i} \Gamma\left(1-\beta_{i 2}^{\prime}-\gamma_{i 2}^{\prime}\right)}{\Gamma\left(1+\alpha_{i}-\beta_{i 2}^{\prime}-\gamma_{i 2}^{\prime}\right)}\left(t-t_{0}\right)^{-\left(\beta_{i 2}^{\prime}-\alpha_{i}\right)} \\
& \quad \leq \frac{\left|x_{i}^{0}\right|}{\Gamma\left(\alpha_{i}\right)} \tilde{T}_{2}^{\frac{1}{2}\left(\alpha_{i}-1\right)}+\frac{l_{i} \Gamma\left(1-\beta_{i 2}^{\prime}-\gamma_{i 2}^{\prime}\right)}{\Gamma\left(1+\alpha_{i}-\beta_{i 2}^{\prime}-\gamma_{i 2}^{\prime}\right)} \tilde{T}_{2}^{-\left(\beta_{i 2}^{\prime}-\alpha_{i}\right)} \leq 1 .
\end{aligned}
$$

Thus, $\left|x_{i}(t)\right| \leq\left[\frac{\left|x_{i}^{0}\right|}{\Gamma\left(\alpha_{i}\right)}\left(t-t_{0}\right)^{\frac{1}{2}\left(\alpha_{i}-1\right)}+\frac{l_{i} \Gamma\left(1-\beta_{i 2}^{\prime}-\gamma_{i 2}^{\prime}\right)}{\Gamma\left(1+\alpha_{i}-\beta_{i 2}^{\prime}-\gamma_{i 2}^{\prime}\right)}\left(t-t_{0}\right)^{-\left(\beta_{i 2}^{\prime}-\alpha_{i}\right)}\right]\left(t-t_{0}\right)^{-\gamma_{i 2}^{\prime}} \leq\left(t-t_{0}\right)^{-\gamma_{i 2}^{\prime}}$ for all $t \geq t_{0}+\tilde{T}_{2}$ and $i=1,2, \ldots, k$. This implies that $x(t)=\left(x_{1}(t), x_{2}(t), \ldots, x_{k}(t)\right) \in S_{2}$, for $t \geq$ $t_{0}+\tilde{T}_{2}$. Since all elements of the set $S_{2}^{\prime}$ tend to 0 as $t \rightarrow \infty$, the zero solution of the problem (2.2) is globally attractive.

\section{Examples}

Here, we give an example to illustrate our results.

Example 4.1 Consider the 3-dimensional system of fractional functional differential equations

$$
\begin{cases}{ }^{c} D^{\frac{1}{2}} x_{1}(t)=\frac{\Gamma\left(\frac{3}{4}\right)}{\Gamma\left(\frac{1}{4}\right)}(t+3) \frac{-3}{4} \frac{\sin ^{2}\left(x_{1}(t-1)\right)}{1+\left(x_{2}(t-1)\right)^{2}} \times \frac{x_{3}(t-1)}{1+\left|x_{3}(t-1)\right|}, & t>0, \\ { }^{c} D^{\frac{1}{4}} x_{2}(t)=\frac{\Gamma\left(\frac{3}{8}\right)}{\Gamma\left(\frac{1}{8}\right)}\left(t+\frac{3}{2}\right)^{\frac{-7}{8}} \frac{\cos ^{4}\left(x_{1}(t-1)\right)}{1+\sin ^{2}\left(x_{3}(t-1)\right)+\left|x_{2}(t-1)\right|}, & t>0, \\ { }^{c} D^{\frac{1}{3}} x_{3}(t)=\frac{\Gamma\left(\frac{5}{6}\right)}{\sqrt{\pi}}(t+1) \frac{-1}{\frac{1}{2}} \frac{\left(x_{1}(t-1)\right)^{4}}{1+\left(x_{1}(t-1)\right)^{4}+6\left|x_{2}(t-1)\right|^{3}}, & t>0, \\ x_{i}(t)=t, \quad i=1,2,3, t \in[-1,0] . & \end{cases}
$$

Define the maps

$$
\begin{aligned}
& f_{1}\left(t, x_{1_{t}}, x_{2 t}, x_{3_{t}}\right)=\frac{\Gamma\left(\frac{3}{4}\right)}{\Gamma\left(\frac{1}{4}\right)}(t+3)^{\frac{-3}{4}} \frac{\sin ^{2}\left(x_{1}(t-1)\right)}{1+\left(x_{2}(t-1)\right)^{2}} \times \frac{x_{3}(t-1)}{1+\left|x_{3}(t-1)\right|}, \\
& f_{2}\left(t, x_{1_{t}}, x_{2_{t}}, x_{3_{t}}\right)=\frac{\Gamma\left(\frac{3}{8}\right)}{\Gamma\left(\frac{1}{8}\right)}\left(t+\frac{3}{2}\right)^{\frac{-7}{8}} \frac{\cos ^{4}\left(x_{1}(t-1)\right)}{1+\sin ^{2}\left(x_{3}(t-1)\right)+\left|x_{2}(t-1)\right|}, \\
& f_{3}\left(t, x_{1_{t}}, x_{2_{t}}, x_{3_{t}}\right)=\frac{\Gamma\left(\frac{5}{6}\right)}{\sqrt{\pi}}(t+1)^{\frac{-1}{2}} \frac{\left(x_{1}(t-1)\right)^{4}}{1+\left(x_{1}(t-1)\right)^{4}+6\left|x_{2}(t-1)\right|^{3}}
\end{aligned}
$$


Baleanu et al. Journal of Inequalities and Applications 2014, 2014:31

Page 12 of 14

http://www.journalofinequalitiesandapplications.com/content/2014/1/31

and put $m_{1}(t)=\frac{\Gamma\left(\frac{3}{4}\right)}{\Gamma\left(\frac{1}{4}\right)}(t+3)^{\frac{-3}{4}}, m_{2}(t)=\frac{\Gamma\left(\frac{3}{8}\right)}{\Gamma\left(\frac{1}{8}\right)}\left(t+\frac{3}{2}\right)^{\frac{-7}{8}}$ and $m_{3}(t)=\frac{\Gamma\left(\frac{5}{6}\right)}{\sqrt{\pi}}(t+1)^{\frac{-1}{2}}$. It is easy to check that $\left|f_{1}\left(t, x_{1_{t}}, x_{2_{t}}, x_{3_{t}}\right)\right| \leq m_{1}(t),\left|f_{2}\left(t, x_{1_{t}}, x_{2_{t}}, x_{3_{t}}\right)\right| \leq m_{2}(t)$ and $\left|f_{3}\left(t, x_{1_{t}}, x_{2_{t}}, x_{3_{t}}\right)\right| \leq$ $m_{3}(t)$. Since

$$
\begin{aligned}
\frac{1}{\Gamma\left(\alpha_{1}\right)} \int_{t_{0}}^{t}(t-s)^{\alpha_{1}-1} m_{1}(s) d s & =\frac{1}{\Gamma\left(\frac{1}{2}\right)} \int_{0}^{t}(t-s)^{-\frac{1}{2}} \times \frac{\Gamma\left(\frac{3}{4}\right)}{\Gamma\left(\frac{1}{4}\right)}(s+3)^{-\frac{3}{4}} d s \\
& \leq \frac{\Gamma\left(\frac{3}{4}\right)}{\Gamma\left(\frac{1}{2}\right) \Gamma\left(\frac{1}{4}\right)} \int_{0}^{t}(t-s)^{-\frac{1}{2}} s^{-\frac{3}{4}} d s=t^{-\frac{1}{4}}, \\
\frac{1}{\Gamma\left(\alpha_{2}\right)} \int_{t_{0}}^{t}(t-s)^{\alpha_{2}-1} m_{2}(s) d s & =\frac{1}{\Gamma\left(\frac{1}{4}\right)} \int_{0}^{t}(t-s)^{-\frac{3}{4}} \times \frac{\Gamma\left(\frac{3}{8}\right)}{\Gamma\left(\frac{1}{8}\right)}\left(s+\frac{3}{2}\right)^{-\frac{7}{8}} d s \\
& \leq \frac{\Gamma\left(\frac{3}{8}\right)}{\Gamma\left(\frac{1}{4}\right) \Gamma\left(\frac{1}{8}\right)} \int_{0}^{t}(t-s)^{-\frac{3}{4}} s^{-\frac{7}{8}} d s=t^{-\frac{5}{8}}
\end{aligned}
$$

and

$$
\begin{aligned}
\frac{1}{\Gamma\left(\alpha_{3}\right)} \int_{t_{0}}^{t}(t-s)^{\alpha_{3}-1} m_{3}(s) d s & =\frac{1}{\Gamma\left(\frac{1}{3}\right)} \int_{0}^{t}(t-s)^{-\frac{2}{3}} \times \frac{\Gamma\left(\frac{5}{6}\right)}{\sqrt{\pi}}(s+1)^{-\frac{1}{2}} d s \\
& \leq \frac{\Gamma\left(\frac{5}{6}\right)}{\Gamma\left(\frac{1}{3}\right) \sqrt{\pi}} \int_{0}^{t}(t-s)^{-\frac{2}{3}} s^{-\frac{1}{2}} d s=t^{-\frac{1}{6}},
\end{aligned}
$$

we get $\left|\frac{1}{\Gamma\left(\alpha_{1}\right)} \int_{0}^{t}(t-s)^{-\frac{1}{2}} f_{1}\left(s, x_{1_{s}}, x_{2_{s}}, x_{3_{s}}\right) d s\right| \leq t^{-\frac{1}{4}},\left|\frac{1}{\Gamma\left(\alpha_{2}\right)} \int_{0}^{t}(t-s)^{-\frac{3}{4}} f_{2}\left(s, x_{1_{s}}, x_{2_{s}}, x_{3_{s}}\right) d s\right| \leq t^{-\frac{5}{8}}$ and $\left|\frac{1}{\Gamma\left(\alpha_{3}\right)} \int_{0}^{t}(t-s)^{-\frac{2}{3}} f_{1}\left(s, x_{1_{s}}, x_{2_{s}}, x_{3_{s}}\right) d s\right| \leq t^{-\frac{1}{6}}$. Now, let $\alpha_{11}=\frac{1}{4}, \alpha_{21}=\frac{1}{8}$ and $\alpha_{31}=\frac{1}{6}$. Then,

$$
\begin{aligned}
\int_{t_{0}}^{\infty}\left|f_{1}\left(t, x_{1_{t}}, x_{2_{t}}, x_{3_{t}}\right)\right|^{\frac{1}{\alpha_{11}}} d t & \leq \int_{t_{0}}^{\infty}\left(m_{1}(t)\right)^{\frac{1}{\alpha_{11}}} d t \\
& =\int_{0}^{\infty}\left[\frac{\Gamma\left(\frac{3}{4}\right)}{\Gamma\left(\frac{1}{4}\right)}(t+3)^{-\frac{3}{4}}\right]^{4} d t \\
& =\frac{1}{18}\left(\frac{\Gamma\left(\frac{3}{4}\right)}{\Gamma\left(\frac{1}{4}\right)}\right)^{4}, \\
\int_{t_{0}}^{\infty}\left|f_{2}\left(t, x_{1_{t}}, x_{2_{t}}, x_{3_{t}}\right)\right|^{\frac{1}{\alpha_{21}}} d t & \leq \int_{t_{0}}^{\infty}\left(m_{2}(t)\right)^{\frac{1}{\alpha_{21}}} d t \\
& =\int_{0}^{\infty}\left[\frac{\Gamma\left(\frac{3}{8}\right)}{\Gamma\left(\frac{1}{8}\right)}\left(t+\frac{3}{2}\right)^{-\frac{7}{8}}\right]^{8} d t \\
& =\left(\frac{2^{5}}{3^{7}}\right)\left(\frac{\Gamma\left(\frac{3}{8}\right)}{\Gamma\left(\frac{1}{8}\right)}\right)^{8}
\end{aligned}
$$

and

$$
\begin{aligned}
\int_{t_{0}}^{\infty}\left|f_{3}\left(t, x_{1_{t}}, x_{2_{t}}, x_{3_{t}}\right)\right|^{\frac{1}{\alpha_{31}}} d t & \leq \int_{t_{0}}^{\infty}\left(m_{3}(t)\right)^{\frac{1}{\alpha_{31}}} d t \\
& =\int_{0}^{\infty}\left[\frac{\Gamma\left(\frac{5}{6}\right)}{\sqrt{\pi}}(t+1)^{-\frac{1}{2}}\right]^{6} d t \\
& =\frac{1}{2}\left(\frac{\Gamma\left(\frac{5}{6}\right)}{\sqrt{\pi}}\right)^{6} .
\end{aligned}
$$


Thus, all conditions of Theorem 3.1 hold and so this system of fractional functional differential equations has an attractive solution.

Example 4.2 Let $0<\alpha_{i}<1, M_{i}>0, \alpha_{i}<\beta_{i 1}^{\prime}<1$ and $x_{i}^{0}$ be a constant for $i=1,2$, 3. Consider the 3 -dimensional system of fractional differential equations

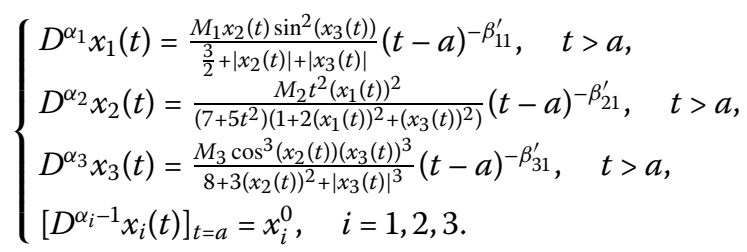

Define the maps

$$
\begin{aligned}
& g_{1}\left(t, x_{1}(t), x_{2}(t), \ldots, x_{k}(t)\right)=\frac{M_{1} x_{2}(t) \sin ^{2}\left(x_{3}(t)\right)}{\frac{3}{2}+\left|x_{2}(t)\right|+\left|x_{3}(t)\right|}(t-a)^{-\beta_{11}^{\prime}} \\
& g_{2}\left(t, x_{1}(t), x_{2}(t), \ldots, x_{k}(t)\right)=\frac{M_{2} t^{2}\left(x_{1}(t)\right)^{2}}{\left(7+5 t^{2}\right)\left(1+2\left(x_{1}(t)\right)^{2}+\left(x_{3}(t)\right)^{2}\right)}(t-a)^{-\beta_{21}^{\prime}}
\end{aligned}
$$

and

$$
g_{3}\left(t, x_{1}(t), x_{2}(t), \ldots, x_{k}(t)\right)=\frac{M_{3} \cos ^{3}\left(x_{2}(t)\right)\left(x_{3}(t)\right)^{3}}{8+3\left(x_{2}(t)\right)^{2}+\left|x_{3}(t)\right|^{3}}(t-a)^{-\beta_{31}^{\prime}} .
$$

Thus, one can check that all conditions of Theorem 3.4 hold and so this system of fractional differential equations has a globally attractive solution.

\section{Conclusions}

Investigating the attractive solutions of the problems is an interesting topic within the fractional calculus. In this manuscript, we focus on the attractivity of solutions for two $k$-dimensional systems of fractional differential equations. Two illustrative examples show the applicability of the proposed methods. The techniques of the reported results can be applied for investigating the attractivity and global attractivity of solutions of different systems of (singular) fractional differential equations. Also, it is an interesting issue to investigate the attractivity and global attractivity of solutions of some systems of fractional differential inclusions.

Competing interests

The authors declare that they have no competing interests regarding the publication of this article.

Authors' contributions

All authors read and approved the final version of this manuscript.

\section{Author details}

'Department of Mathematics, Cankaya University, Ogretmenler Cad. 14, Balgat, Ankara, 06530, Turkey. ${ }^{2}$ Institute of Space Sciences, Magurele, Bucharest, Romania. ${ }^{3}$ Department of Chemical and Materials Engineering, Faculty of Engineering, King Abdulaziz University, P.O. Box 80204, Jeddah, 21589, Saudi Arabia. ${ }^{4}$ Department of Mathematics, Azarbaijan Shahid Madani University, Azarshahr, Tabriz, Iran.

\section{Acknowledgements}

Research of the second and third authors was supported by Azarbaijan Shahid Madani University. Also, the authors express their gratitude to the referees for their helpful suggestions which improved the final version of this paper. 


\section{References}

1. Ahmad, B: Existence of solutions for irregular boundary value problems of nonlinear fractional differential equations. Appl. Math. Lett. 23, 390-394 (2010)

2. Ahmad, B, Nieto, JJ: Existence results for a coupled system of nonlinear fractional differential equations with three-point boundary conditions. Comput. Math. Appl. 58, 1838-1843 (2009)

3. Ahmad, B, Sivasundaram, S: Existence results for nonlinear impulsive hybrid boundary value problems involving fractional differential equations. Nonlinear Anal. 3, 251-258 (2009)

4. Ahmad, B, Sivasundaram, S: Existence of solutions for impulsive integral boundary value problems of fractional order. Nonlinear Anal. 4, 134-141 (2010)

5. Agarwal, RP, Andrade, B, Cuevas, C: Weighted pseudo-almost periodic solutions of a class of semilinear fractional differential equations. Nonlinear Anal. 11, 3532-3554 (2010)

6. Agarwal, RP, Benchohra, M, Hamani, S: A survey on existence results for boundary value problems of nonlinear fractional differential equations and inclusions. Acta Appl. Math. 109, 973-1033 (2010)

7. Agarwal, RP, Lakshmikantham, V, Nieto, JJ: On the concept of solution for fractional differential equations with uncertainty. Nonlinear Anal. 72, 2859-2862 (2010)

8. Balachandran, K, Kiruthika, S: Existence of solutions of abstract fractional impulsive semilinear evolution equations. Electron. J. Qual. Theory Differ. Equ. 2010, 4 (2010)

9. Bai, C: Existence of positive solutions for boundary value problems of fractional functional differential equations. Electron. J. Qual. Theory Differ. Equ. 2010, 30 (2010)

10. Baleanu, D, Agarwal, RP, Mohammadi, H, Rezapour, S: Some existence results for a nonlinear fractional differential equation on partially ordered Banach spaces. Bound. Value Probl. 2013, 112 (2013)

11. Baleanu, D, Mohammadi, H, Rezapour, S: Positive solutions of an initial value problem for nonlinear fractional differential equations. Abstr. Appl. Anal. 2012 Article ID 837437 (2012)

12. Baleanu, D, Mohammadi, H, Rezapour, S: Some existence results on nonlinear fractional differential equations. Philos. Trans. R. Soc. Lond. A 371, 20120144 (2013)

13. Baleanu, D, Mohammadi, H, Rezapour, S: On a nonlinear fractional differential equation on partially ordered metric spaces. Adv. Differ. Equ. 2013, 83 (2013)

14. Burton, TA: A fixed point theorem of Kranoselskii. Appl. Math. Lett. 11, 85-88 (1998)

15. Chen, F, Nieto, JJ, Zhou, Y: Global attractivity for nonlinear fractional differential equations. Nonlinear Anal. 13, 287-298 (2012)

16. Chen, F, Zhou, Y: Attractivity of fractional functional differential equations. Comput. Math. Appl. 62, 1359-1369 (2011)

17. Hale, JK: Theory of Functional Differential Equations. Springer, Berlin (1977)

18. Kilbas, AA, Srivastava, HM, Trujillo, JJ: Theory and Applications of Fractional Differential Equations. North-Holland Mathematics Studies, vol. 204. Elsevier, Amsterdam (2006)

19. Kranoselskii, MA: Topological Method in the Theory of Nonlinear Integral Equations. Macmillan Co., New York (1964)

20. Tian, Y, Bai, Z: Existence results for the three-point impulsive boundary value problem involving fractional differential equations. Comput. Math. Appl. 59, 2601-2609 (2010)

21. Wang, G, Ahmad, B, Zhang, L: Impulsive anti-periodic boundary value problem for nonlinear differential equations of fractional order. Nonlinear Anal. 74, 792-804 (2011)

22. Zhou, Y, Jiao, F, Li, J: Existence and uniqueness for fractional neutral differential equations with infinite delay. Nonlinear Anal. 71, 3249-3256 (2009)

23. Pinto, C, Machado, JAT: Forced van der Pol oscillator of complex order. In: ENOC 2011, Rome, Italy, 24-29 July (2011)

24. Rihan, FA: Numerical modeling of fractional order biological systems. Abstr. Appl. Anal. 2013, Article ID 816803 (2013)

25. Suchorsky, MK, Rand, RH: A pair of van der Pol oscillators coupled by fractional derivatives. Nonlinear Dyn. 69 313-324 (2012)

10.1186/1029-242X-2014-31

Cite this article as: Baleanu et al.: Attractivity for a $k$-dimensional system of fractional functional differential equations and global attractivity for a $k$-dimensional system of nonlinear fractional differential equations. Journal of Inequalities and Applications 2014, 2014:31

\section{Submit your manuscript to a SpringerOpen ${ }^{0}$ journal and benefit from:}

- Convenient online submission

- Rigorous peer review

- Immediate publication on acceptance

- Open access: articles freely available online

- High visibility within the field

- Retaining the copyright to your article 